\title{
Social and individual learning of helping in humans and other species
}

\author{
Laurent Lehmann ${ }^{1}$, Kevin R. Foster ${ }^{2}$, Elhanan Borenstein ${ }^{1}$ and Marcus W. Feldman ${ }^{1}$ \\ ${ }^{1}$ Department of Biology, Stanford University, Stanford, CA 94305, USA \\ ${ }^{2}$ Bauer Center for Genomics Research, Harvard University, Cambridge, MA 02138, USA
}

\begin{abstract}
Helping behaviors can be innate, learned by copying others (cultural transmission) or individually learned de novo. These three possibilities are often entangled in debates on the evolution of helping in humans. Here we discuss their similarities and differences, and argue that evolutionary biologists underestimate the role of individual learning in the expression of helping behaviors in humans.
\end{abstract}

\section{Ontogeny of helping behaviors}

Helping and cooperative behaviors, whereby individuals in a population increase the fitness of or payoff to other individuals, is widespread in the natural world, both across taxa and at different levels of biological organization (see Glossary for a definition of terms). Examples range from interactions between genes in genomes to mutualism between species in ecological communities. Among this diversity, one of the most impressive examples of cooperation occurs in humans. At the heart of human sociality are behaviors and actions in which one individual provides help for another. These behaviors have fascinated scholars for centuries [1-4], and a contemporary of Darwin, Augustus Comte, introduced the term 'altruism' in an attempt to capture their essence [5]. Comte's work, which focused on intentionality - whether an individual intends to do good by an action-formed the foundation for the humanities' interest in altruism. Ever since Darwin, however, biologists have cared more about the evolutionary outcome of actions than what was intended, and William D. Hamilton's foundational analysis led to the distinction between two types of helping behaviors [4,6,7]. First, a helper can reduce its own fitness or payoff, but the act of helping is rewarded by the prospect of immediate or future return due to helping by another individual in the population. Reciprocation is expected and the lifetime fitness of the actor is increased. Second, helping can result in a net reduction in the lifetime fitness to the helper. In this case, helping is said to be altruistic sensu stricto $[4,8,9]$

Understanding the variation in the prevalence and nature of helping behaviors in humans and other species requires identifying all the factors causing their evolution, as well as those determining their expression during the lifetime of an individual [10]. That is, one must be careful to distinguish between ultimate and proximate causes of behaviors. Ethologists tend to divide proximate causes into two categories (e.g. [11-13]). There are motivations, which

Corresponding author: Lehmann, L. (lehmann@stanford.edu). are the stimuli causing the expression of a behavior at a given moment in time, and ontogeny, which refers to the determinants of the behavior map; namely, the causes defining the relationship between stimuli (e.g. hunger) and the organism's behavioral response (e.g. searching for food) (see Figure I in Box 1). What then determines whether an individual will produce a cooperative response to a particular set of stimuli? In other words, what determines the behavior map of helping behaviors? An evolutionary biologist will often first consider that a helping behavior might be genetically determined. However, an emphasis on simple genetics can be misleading. Indeed, behaviors can be transmitted not only genetically from parent to offspring, but also culturally from one individual to another in a population. Another factor determining the behavior map of helping is therefore social learning, by which an individual can copy or adopt the behavior of another in a population [11,12]. Birds learn song dialects from neighbors and chimpanzees the skills of 'termiting' with tools $[11,12]$, and it is plausible that animals that

\section{Glossary}

Altruism: a behavior that increases the fitness of another individual but decreases the fitness of the actor.

Behavior map: functional relation between the external and internal states of an organism (collectively called the motivational states) and its behavior.

Conformist transmission: individual preference for adopting common cultural traits by social learning.

Cooperation: a behavior that increases the fitness of another individual. Cultural relatedness: measure of the tendency that a pair of interacting individuals is more likely to carry identical cultural variants than is a pair of individuals sampled at random from a population.

Cultural transmission: nongenetic transmission of information or of a trait from one individual to another in a population.

Darwinian demon: hypothetical being with the power to decide exactly which phenotype to express, but without any influence over the ensuing natural selection.

Evolutionary stable strategy: a strategy which, if adopted by a population of players, cannot be invaded by any alternative strategy that is initially rare.

Fitness: expected number of adult offspring produced by an individual.

Genetic relatedness: measure of the tendency that a pair of interacting individuals is more likely to carry identical genetic variants (alleles) than is a pair of individuals sampled at random from a population.

Helping: a behavior that increases the fitness of another individual.

Individual learning: psychological (cognitive) processes supporting the generation or the innovation of behaviors that were not transmitted genetically or culturally.

Payoff: a reward for the action of an individual.

Proximate factors: explanations of a behavior referring to the motivational state of an organism and the factors determining the ontogeny of the behavior (genotype and experience).

Social learning: psychological (cognitive) processes supporting cultural transmission of behaviors.

Ultimate factors: explanations of a behavior referring to selection pressure and other factors that cause the evolution of the behavior. 


\section{Box 1. Behavior map of helping behaviors}

Ethologists often assume that the behavior of an organism can be predicted from knowledge about a set of external stimuli and internal states of the organism (e.g. $[13,89])$. Together these are called the set of motivational states, and are proximate explanations of behavior. To each set $\mathbf{s}$ of motivational states of an animal at a given moment in time one can assign a behavioral response $z$. Mathematically, this is expressed as

$z=M(s)$

where $M$ is the behavior map defining the relationship between $s$ and $z$ (Ref. [13], Equation 1.1). This approach to behavior also encompasses social behaviors, where the behavior map represents the action taken by an organism at a given moment in time when interacting with other individuals (e.g. Ref. [33] and references therein). For instance, Tit-forTat can be represented by a behavior map in which the motivational state $s$ can be taken to be the move of the partner in the preceding round (either $C$ for cooperation or $D$ for defection); then, $M=C$ on the first move and $M(s)=s$ for subsequent moves (e.g. $M[D]=D$ and $M[C]=C)$.
The behavior map $M$ of an individual might be determined by its genotype $g$ and/or what it has learned, $h$, either through social interactions or individual exploration ( $h$ can be thought of as the learning history of the individual). This can be expressed as

$M=F(g, h)$,

where $F$ describes how the behavior map used by an individual at a given moment in time is determined by its genes and its learning history (see Figure I for a complementary view), which provide a second type of proximate explanations of behaviors. For instance, the behavior map for Tit-for-Tat can be determined genetically, culturally, learned individually or some combination of these factors (Figure I).

The learning history $h\left(e, l_{i}, l_{s}\right)$ of an individual depends on $e$, the history of the individual's environment, its social learning rule, $l_{s}$, and its individual learning rule, $l_{i}$, used to obtain information from the environment. Finally, the two learning rules used by an individual during its lifetime when interacting with its environment are shaped by the individual's genotype, $\mathrm{g}$, and some aspects of its environment, e (i.e. $I_{s}[g, e]$ and $l_{i}[g, e]$; see Figure $\left.I\right)$.

(a)

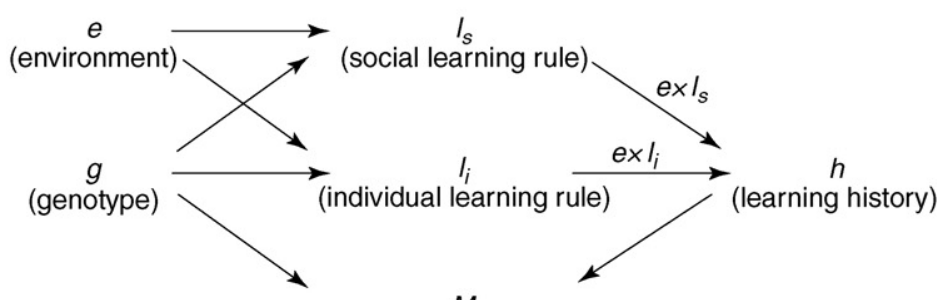

$\stackrel{M}{\text { (behavior map, e.g. Tit-for-tat) }}$

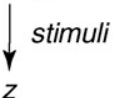

(behavior)

(b)

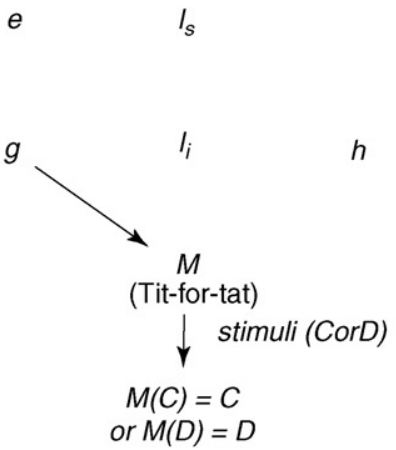

(c)

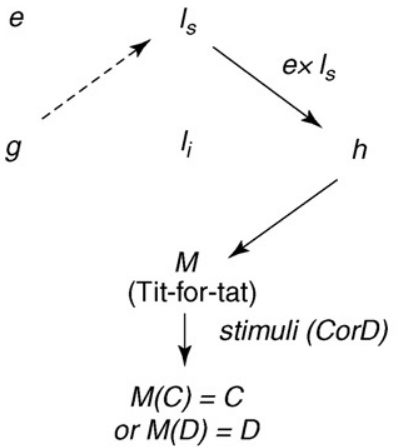

(d)

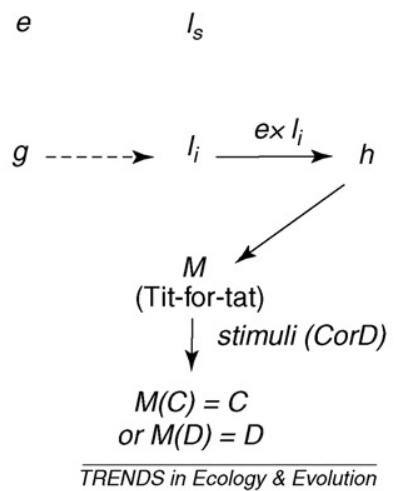

Figure I. Figurative representation of the determinants of the behavior map as described in Box 1 . (a) Generic representation. The genotype, $g$, and the environment, $e$ of an individual determine its social and individual learning rules $\left(I_{s}\right.$ and $\left.I_{i}\right)$. These, in interaction with the environment (depicted as $e \times I_{s}$ and $e \times I_{i}$ in the figure), determine the learning history, $h$, of the individual (i.e. its experience). The genotype can affect the behavior map, $M$. either directly or indirectly via the learning dynamics. (b-d) Determinants of the behavior map Tit-for-tat as they are generally considered in models for the evolution of cooperation. (b) The behavior map is entirely genetically determined (e.g. [25]). (c) The behavior map is socially learned and it is implicitly assumed that individuals can perform social learning (e.g. [43]); that is, the evolution of the social learning rules $I_{s}$ and their genetic foundation are not explicitly considered but assumed implicitly (hence the dashed arrow for the effects of the genotype on the social learning rule $I_{s}$ ). (d) The behavior map is individually learned (e.g. [66]). Again, the evolution of the individual learning rules $l_{i}$ are not explicitly considered. In existing models, there is also no effect of the environment on the learning rules, but the learning history depends on the interaction between the expression of the social and individual learning rules and the environment $\left(e \times I_{s}\right.$ and $e \times I_{i}$ in the figure).

learn socially also copy helping behaviors from others. There is yet a third determinant of behavior maps, more familiar to economists than biologists in the context of helping: individual learning of cooperation $[14,15]$. Individual learning of a behavior is the relatively permanent change of an individual's behavior during the lifetime of that organism, without copying or imitating others. Animals might learn several things individually, from food to mates to predators $[11,12]$, and it is plausible that they also learn individually to cooperate or defect. Genetic, cultural transmission and individual learning of helping behaviors are dynamical systems that are all likely to 
affect the expressions and evolution of cooperation in humans and some other species. Whereas these interlinked processes are well recognized in the study of animal behavior by biologists [12] and the surveys of human behavior by economists $[14,16]$, they seldom appear together in writings on the evolution of human cooperation. We believe that, as a result, several biases have crept into the literature. Our goal here is to discuss the similarities and the differences between genetic, cultural transmission and individual learning of helping behaviors, and ask: which is likely to promote the highest level of helping and is, therefore, the most important for human cooperation?

\section{Genetic transmission of helping behaviors}

Evolutionary biologists typically model the behavior map of both simple and complex helping strategies as genetically determined (e.g. $[4,17,18]$ ). For example, the worker caste in social insects and the provision of a public good, such as a shared product, in microorganisms are genetically inherited phenotypes (e.g. [19,20]). The ultimate explanation for such helping behaviors often centers on genetic relatedness between actor and recipient $[4,8,17]$. When actor and recipient are related, genetically determined altruistic helping can evolve because the benefit produced by a helper is likely to be received by another individual carrying the gene(s) causing helping, instead of being received by an individual carrying the gene(s) for less or no helping. One of the main questions is then to understand how ecological, demographic and lifehistory factors interact to generate an assortment between individuals carrying genes for altruism. Many different models have addressed this issue (e.g. [8,9,17,21-23]), and a general conclusion from these is that for altruism to evolve, the group size of interacting individuals must not be too large and/or the rate of migration not too high, otherwise relatedness within groups becomes too small to sustain the evolution of altruism. A genetically determined behavior map is then a good candidate to explain the altruism found within small-scale societies. Humans, however, have also been documented to reduce their personal reproduction in helping unrelated individuals (e.g. religious celibacy, kamikaze behavior), and in such cases other proximate determinants of behaviors than genetic must be invoked to explain such behaviors.

Not all helping behaviors, however, reduce personal reproduction. When interactions occur between individuals sampled at random from a population (i.e. a well-mixed population with no relatedness between actor and recipient), a genetically determined helping strategy might evolve if it is rewarded by the prospect of immediate or future cooperation by the partner. This observation led evolutionists to model the interactions between individuals as games with repeated interactions [24,25], as had been done previously by economists (the so-called multistage and repeated games [16,26-28]). The common underlying principle in such games is that links between current and future actions can create incentives to help that are not apparent if single actions are considered in isolation. The emergence of such incentives are often studied in the context of the iterated prisoner's dilemma game (IPD; Box 2), whose analysis popularized the strategy Tit-for-Tat, which helps in the first round and then adopts the strategy of its partner

\section{Box 2. Terms used in game theory}

Bargaining game: a type of negotiation where there is an exchange of offers between individuals who eventually come to an agreement on the partitioning of a payoff.

Folk theorem of repeated games: result of game theory stipulating that there are an infinite number of Nash equilibria in the iterated prisoner's dilemma game.

Game: any situation where the success of an individual's choices depends on the choices of other individuals.

Iterated prisoner's dilemma (IPD): game where the prisoner's dilemma game is repeated several times. The interdependence of actions between rounds of the game creates incentives to cooperate that are absent in the one-shot prisoner's dilemma game.

Nash equilibrium: equilibrium of a game where no player can increase its payoff by changing its strategy, while the other players keep their strategy unchanged. Each evolutionary stable strategy is a Nash equilibrium, but the converse is not true.

Preference: a notion from economics which assumes that individuals make choices between alternatives and have the possibility to rank order these alternatives, based on the satisfaction, gratification, enjoyment or utility they provide.

Prisoner's dilemma game: a situation where players interact anonymously, only once, and have the choice between two behaviors: cooperate or defect. Independently of the choices of others, the payoff to an individual is always lower when it cooperates than when it defects.

Public goods game: special case of the prisoner's dilemma game where the choices between behavior involve the consumption or the use of a resource common to all players.

Tit-for-Tat: famous strategy in the iterated prisoner's dilemma game. This strategy helps in the first round and thereafter adopts the strategy of its partner from the previous round.

Ultimatum game: a situation where two players interact anonymously and only once. The first player proposes how to divide a sum of money between the two players, and the second player can either accept or reject this proposal. If the second player rejects, neither player receives anything. If the second player accepts, the money is split according to the proposal.

from the previous round [15,25,29]. The outcome of the IPD game, however, can be extremely difficult to predict because there are multiple solutions to it. More precisely, Tit-for-Tat is neither an evolutionary stable strategy [30] nor a strict Nash equilibrium, and there are in fact an infinite number of Nash equilibria in the IPD, a result known as the 'folk theorem' of repeated games [15,31,32]. This explains why evolutionists have analyzed a bewildering number of different models to assess which strategies perform better or coexist in the IPD games and various other games (e.g. [33-38]). These models show that genetically determined responses to repeated interactions will often evolve to produce helping behaviors. Even so, these models lack the social learning observed in humans, and this has led researchers to consider a second mode of inheritance of helping traits: cultural transmission.

\section{Social learning of helping behaviors}

The study of cultural evolution descends directly from existing evolutionary theory [39], with the difference that traits are assumed to be inherited culturally rather than genetically. For instance, a public goods game can be analyzed within an evolutionary setting by assuming cultural rather than genetic determination of behavior maps. But switching to cultural transmission does bring in additional considerations. Whereas genetically determined behaviors tend to follow strict vertical transmission 
from parent to offspring, cultural transmission can also occur obliquely from various adults to individuals of the offspring generation, or horizontally between individuals of the same generation [40-42]. For instance, individuals might copy a helping strategy from their grandparents, or from unrelated members of present and past generations. Another important consideration is what drives the differential proliferation of cultural traits: differential success can result from effects of cultural traits on darwinian (reproductive) fitness [40,43] or, alternatively, from their effects on some other payoff such as wealth or prestige. In the latter case, the dynamics of helping are often assumed to be mediated by individuals copying the strategies of individuals with the highest payoffs (e.g. [43-46]). But does the change from genetic to cultural transmission of helping behaviors produce different evolutionary dynamics of these traits?

When interactions occur among individuals drawn at random from a population, the dynamics of helping strategies under both genetic and cultural transmission often lead to identical outcomes. This similarity occurs in spite of the differences between genetic and cultural transmission rules. A rich variety of more or less sophisticated transmission rules have been explored under social learning (e.g. 'best-response dynamics' or 'fictitious play' [43,47,48] and references therein), but, because identical behaviors lead to identical payoff values, there are similar selective pressures for genetically and culturally transmitted traits. As a result, economists often adopt the classical equations from population genetics to describe the evolutionary dynamics of various strategies (e.g. [49-51]), and many of the genetic models for repeated interactions discussed in the previous section are presented as being equally valid for cultural transmission (e.g. [52]).

Although for numerous games many dynamics indeed lead to the same predictions under both genetic and cultural transmission [53], there are properties of cultural transmission that might cause these predictions to diverge. For example, under cultural transmission it might be difficult to observe and copy all the fine details of a complicated strategy, especially because only actions can be observed and not the behavior map of a strategy. It remains to investigate whether such factors will increase or decrease the equilibrium level of cooperation for a given game. Nevertheless, there is one difference between cultural and genetic transmission of helping that has received considerable attention, because it might allow culturally transmitted altruistic helping to evolve where genetic altruism cannot. This distinctive feature is that cultural transmission allows helping behaviors to be easily transmitted among genetically unrelated individuals. Whereas under genetic transmission it is the ecological, demographic and life-history factors that determine the genetic relatedness between interacting individuals, under cultural transmission the cultural relatedness between interacting individuals is determined by how individuals assimilate cultural variants from other individuals in the population. Nonvertical transmission can then homogenize behavior within groups, leading to high cultural relatedness within groups lacking high levels of genetic relatedness $[40,54,55]$. Cultural transmission might there- fore markedly increase the chance that helpers or defectors occur together in a group relative to that expected under genetic transmission. Specifically, it has been argued that humans have a predisposition for conformism - the copying of the majority strategy - which naturally leads to groups where individuals all perform the same strategy $[46,54,56,57]$.

The observation that cultural transmission can increase the assortment between identical strategies has led to two complementary views: first, that cultural transmission is central to the observed levels of altruistic helping in humans and, second, that biased conformism is the main transmission rule underlying such altruism (e.g. $[46,54,57-59]$ and references therein). These conclusions, however, require caveats. The two predictions that cultural transmission enhances the selection pressure on altruism and that conformist transmission is the kernel of the process are based on a specific set of assumptions, which, if violated, can produce the opposite results $[60,61]$. It is premature, therefore, to argue that cultural transmission is the key to human helping behaviors. This requires a far better understanding of the conditions under which

\section{Box 3. Debate: does cultural transmission promote altruism?}

Here we describe the conditions under which cultural transmission leads to a higher level of altruism than under genetic transmission in evolutionary models. Suppose the population is of infinite size and that a strictly one-shot interaction occurs between randomly matched pairs of individuals that can express only two different behaviors: altruist, which results in a fitness cost $c$ to the actor and a fitness benefit $b$ to the receptor, and defector, which causes the actor to take the benefits of helping at no cost. Under genetic transmission with the two behaviors transmitted vertically from parents to offspring, a population consisting entirely of altruists is invaded by defection, which is the classic finding associated with the prisoner's dilemma game. Now suppose we add in a second round of transmission, which is cultural and whereby each individual after pairing adopts the behavior of one of the two that is randomly drawn in the pair (i.e. one-to-many horizontal transmission), with the consequence that both individuals in the pair now carry the behavior. Because such a rule causes perfect assortment of altruists and defectors, altruism can invade a population of defectors. If such a social learning rule exists, the evolution of altruism is more likely under cultural than under pure genetic transmission of the behavior. However, the problem is only half solved because one is now faced with another and equally important question: can such a learning rule evolve when the population is initially fixed for vertical transmission only?

The answer depends on multiple factors. One can show that a genetically determined learning rule in a simple model can indeed evolve, and be stable upon fixation, provided the group of randomly matched individuals is small-such as the pair in our example-and that the learning rule is not itself costly [61]. However, this finding relies on excluding strategies from the model framework that can cause assortment to fail. Indeed, individuals are assumed to be able to copy helping behaviors (from individuals in the same or previous generations), but also that they do not use the information gathered on the cooperative tendency in their social neighborhood to exploit others. This strong assumption is a common denominator of all current models assuming the cultural transmission of altruism and cooperation (e.g. $[44,45,54]$ ). Much work remains to be done to establish the conditions under which cultural transmission would evolve (or be stable) in such a way as to tip the balance in favor of helping in situations under which genetic transmission of behavior maps favors defection. 
cultural inheritance will enhance rather than hinder the evolution of altruistic helping (Box 3).

\section{Individual learning of helping behaviors}

The discussion so far has emphasized the differences between the transmission processes of genetically and culturally encoded behavior maps. It is then the differences in the proliferation of helping strategies from one time span to the next that determine the equilibrium distribution of strategies played by the individuals in the population. However, not all human game-playing strategies are determined only by genetic or cultural transmission; humans can also select a behavior individually, through trial and error, insight or deduction [16]. Here it is not a specific behavior map that is inherited genetically or culturally by an individual but a payoff-based choice-determining rule by which it can modify its behavior map(s) throughout its lifetime by individual exploration (see Figure I in Box 1).

The study of individual learning rules has a long history in economics, psychology and biology (e.g. [13,62-65]), and two nonmutually exclusive classes of strategies can be recognized. The first is exemplified by reinforcement learning, where the probability of playing a particular strategy is adjusted over time in response to the current payoff. This allows an individual to select a strategy for a game without ever observing or knowing anything about other individuals (e.g. [66]). This learning by doing is referred to as the 'evolutive' approach to the individual behavioral choice problem in the economics literature $[32,67,68]$ probably because, as in evolutionary settings, there is a dynamical process of selection by which the most rewarding strategies succeed over those that are less rewarding (e.g. [66,69]). This approach envisages individuals as simple stimulusresponse machines who can select a strategy among a set of alternatives in the absence of a complete understanding of the underlying game structure (hence the term 'bounded rationality' of the economics literature). Here, Tit-for-Tat will be played in the IPD game if it is reinforced over time by a consistently higher payoff than alternative strategies experienced by the actor $[66,69]$.

The second class of payoff-based choice-determining rules relies on individuals having detailed knowledge of the game they are in and using reasoning to find their way toward one of the equilibria of the game. That is, individuals perceive the situation and the consequence of their actions and those of others as a whole, and deduce a response for these. In the economics literature, this is the so-called deductive or eductive approach to the behavioral selection problem $[32,67,68]$. The incarnation of such learning by reasoning is the 'rational man' idealization, a machine with unbounded powers of computation and cogitation [70] which has the ability to simulate its partner simulating its own behavior, to be able to predict what the partner will play (nothing prevents the rational man idealization from using social information). Under the deductive approach to behavioral selection (learning by reasoning), individuals are assumed to select a strategy because it corresponds to a Nash equilibrium of the game they playor some related concept of it-and economists dispute which is the correct equilibrium concept to use $[32,67,68,71]$. In other words, the strategy Tit-for-Tat would be played in the IPD game in this context not because of selection caused by genetic, cultural or behavioral reinforcement dynamics but because individuals deduce that playing Tit-for-Tat is a best response in a population of Tit-for-Tat players.

As for social learning, the predicted dynamics of cooperation under individual learning can show significant overlap with those under genetic transmission. In particular, if a learning rule is evolutionarily stable, it follows that it should allow individuals to find the evolutionary stable strategy of the game played under genetic transmission of the behaviors [62,72], provided there is such an evolutionary stable strategy. Therefore, all else being equal, the equilibrium level of cooperation under individual learning is predicted to be the same as that under genetic transmission. That said, few models have addressed the evolution of individual learning rules for social interactions [65] and, to our knowledge, no study has directly compared the propensity for individual learning to promote cooperation relative to that under social learning or genetic transmission of behavior maps. Such comparisons might prove to be useful. Because a behavior selected by individual learning is not inherited from one generation to the next (in the absence of social learning), such a choice-determining rule has no advantage over genetic transmission in constant environments. But individual learning has the potential to allow individuals to adapt to rapidly changing environments (e.g. $[42,63,72,73])$. That is, individual learning allows a much closer tracking of fluctuations in the environment (whether social and nonsocial environments) than does genetic inheritance. Because humans have evolved under conditions of fluctuating environments and have strong dispositions for individual learning [74], they might arguably also be able to easily modify their social behaviors. But is there any empirical evidence that humans can learn to find an equilibrium of a game they play?

\section{Individual learning of helping in humans}

Many data have been collected on the behavior of humans in economic games, in which researchers bring subjects into the laboratory, explain a set of rules and let the subjects play for monetary rewards. If the problem faced by the subjects is not too complex, and subjects have adequate incentives and ample time to learn, convergence of behavior to the equilibria of games by individuals has been repeatedly documented whenever the game has a single Nash equilibrium [15,75]. For instance, in the public goods game, whose only equilibrium is to contribute nothing, $90 \%$ of subjects end up playing this strategy after several iterations of the game [76,77]. In bargaining games, which are close to real-life trading situations, humans are also able to find the theoretical equilibrium [75]. These results have then been interpreted in the light of individual learning $[15,75]$. However, alternative explanations for the public goods game results based on fixed strategies can be proposed (S. Gaechter, pers. commun.), and there are also instances where behavior deviates from theoretical expectations. For instance, in public goods games, where a focal individual learns to play a Nash equilibrium, removing the individuals that she learned with and replacing 
them with new individuals can lead the focal individual to revert to play a non-Nash equilibrium [78]. Furthermore, in bargaining games, there is a general tendency to refuse inequitable offers [75], a tendency further exaggerated in the ultimatum game, where humans adjust very little through learning and continuously play more fairly than expected [15,79]. These contrasting examples paint a vague picture of how individual learning affects human helping. It remains unclear why subjects play the way they do, and a lot of attention has been paid to those games in which humans play more fairly than expected.

The observation of a much fairer contribution than expected in a subset of experimental games has been explained in the light of two different conceptions of the nature of human helping. One perspective envisions individuals as expressing relatively fixed 'other-regarding' preferences, valuing cooperation, fairness and retribution (e.g. [79-81]). This conception follows from the observation of a behavioral regularity in experimental games that has been called strong reciprocity, a predisposition to cooperate with others and punish non-cooperators (at a cost to the punisher), even if the punishment will never increase the lifetime fitness of the punisher [79,82]. The second perspective views individuals as 'self-regarding' decision makers who aim to maximize their own monetary rewards (or material payoffs) and can find their way toward a maximum reward by learning $[15,16]$. The fair contribution from experimental games is then explained by invoking a learning process with two behavioral time steps. In the first time step, the frame of the experiment (i.e. external stimuli) triggers the expression of a genetically or culturally encoded social norm from a real-life game, which involves, for instance, helping a family or a group member [83]. In a second time step, when the same game is repeated several times with different partners, individual learning might occur. Individual learning is predicted to be very slow in games that are characterized by multiple Nash equilibria [15]. The ultimatum game has an infinite number of such equilibria, only one of which-contributing nothing-is stable in the ultra long run [84]. The social norm triggered initially is thus expected to dominate for a very long time before the learning dynamic finds the longrun equilibrium of the game [84]. These two different proximate explanations for a fair contribution in experimental games illustrates again that the underpinning of behavior maps used by subjects in experiments remains unclear and is much debated, not to mention the effect of the internal states of individuals such as serotonin level [85].

As demonstrated above, debates over the nature of human helping often focus on whether people display other-regarding or self-regarding preferences (e.g. [7981]). This distinction, however, is based upon the observation of behavioral preferences rather than their explanation (the 'revealed preference' principle), which means that it does not translate directly into an evolutionary accounting. Observed preferences-other-regarding or otherwise-depend on how behavior maps transform stimuli into behavioral responses, and the mappings that persist in the long run are those that increase the frequency in the population of the genetic or the cultural variants causing them [86]. Moreover, both other-regarding and self-regarding preferences are likely to frequently involve direct benefits to the individuals that display them once all lifetime behavioral feedbacks from their expression have been taken into account, that is, 'other-regarding' does not map onto altruism defined in the strict evolutionary sense (i.e. Hamilton's altruism). Where the two perspectives differ, however, is in their relative emphasis on inherited versus individually learned mappings: the literature on other-regarding preferences tends to treat individuals as irreversibly committed by genetic or cultural helping variants to express a fixed pattern of behaviors. The self-regarding literature, by contrast, suggests a highly plastic behavior map, which can be continually adjusted in a way that yields the actors the highest payoff in each new game [15], probably the closest we can get to a darwinian demon [87]. Rather than focusing on other- or self-regarding preferences of individuals, therefore, we believe that the debate over the nature of human helping would be more usefully focused on the effects and relative importance of behavioral plasticity, whether socially or individually learned.

\section{Conclusion}

Behavior maps, by which an organism translates external stimuli and internal states into action, can be genetically determined, cultural transmitted, individually learned or some combination of these factors. Understanding the structure and evolution of the behavior maps underlying human cooperation and altruism remains a daunting challenge. Today, few will doubt the importance of genetic influence on human behavior. Nevertheless, it remains plausible that the human capacity to learn and personal experience are more central to the ontogeny of helping than direct genetic effects on behavior maps (see Figure I in Box 1). This is consistent with the growing body of theory on social learning of helping behaviors, which identifies conditions under which altruism can evolve in groups of large size where individuals engage in cultural transmission (e.g. $[57,88])$. However, the same theory can be used to find conditions under which cultural transmission inhibits altruism, and we must not be too quick to assume that cultural transmission promotes helping (Box 3). Furthermore, the literature on social learning of cooperation tends to give short shrift to learning by doing or by reasoning, processes that allow a much faster response to changing environmental conditions than genetic mutation and social learning. The role of individual learning is thus likely to have been of equal importance to that of social learning in the expression of cooperation throughout human history. The nature of this role remains to be elucidated, as does the question of whether individual learning promotes or inhibits the evolution of cooperation. As for social learning, more empirical and theoretical work are required to better understand the effects of individual learning of helping behavior and its interaction with cultural transmission. The difficulty of the problem is compounded by the fact that learning individuals might not only adapt to a particular game defined by prevailing environmental conditions but might also change or design the rules of the game they play (i.e. evolution of games of mechanism design $[28,48]$ ). This 
raises the intriguing question of how often and how early humans were able to shape the game of life in such a way as to promote the expression of more cooperative solutions.

\section{Acknowledgements}

We thank Jeremy Van Cleve, Anna Dreber, Martine Ehinger and Christopher Hauert for useful comments on previous versions of this manuscript, and Edith Katsnelson for providing references. L.L. is supported by a grant from the Swiss NSF. K.R.F. is supported by National Institute of General Medical Sciences Center of Excellence grant 5P50 GM 068763-01. E.B.'s research is supported in part by the Morrison Institute for Population and Resource Studies, a grant to the Santa $\mathrm{Fe}$ Institute from the James S. McDonnell Foundation 21st Century Collaborative Award Studying Complex Systems and by NIH grant GM28016. This research is supported by NIH grant GM28016.

\section{References}

1 Smith, A. (1776) The Wealth of Nations, Penguin Classics

2 Darwin, C.R. (1859) On the Origin of Species by Means of Natural Selection: Or the Preservation of Favoured Races in the Struggle for Life, John Murray

3 Haldane, J.B.S. (1932) The Causes of Evolution, Princeton University Press

4 Hamilton, W.D. (1964) The genetical evolution of social behaviour, I. J. Theor. Biol. 7, 1-16

5 Dixon, T. (2008) The Invention of Altruism: Making Moral Meanings in Victorian Britain, Oxford University Press

6 Hamilton, W.D. (1970) Selfish and spiteful behavior in an evolutionary model. Nature 228, 1218-1220

7 Hamilton, W.D. (1971) Selection of selfish and altruistic behaviour in some extreme models. In Man and Beast: Comparative Social Behavior (Eisenberg, J. and Dillon, W., eds), pp. 59-91, Smithsonian Institution Press

8 Grafen, A. (1985) A geometric view of relatedness. In Oxford Surveys in Evolutionary Biology (Dawkins, R. and Ridley, M., eds), pp. 28-90, Oxford University Press

9 Rousset, F. (2004) Genetic Structure and Selection in Subdivided Populations, Princeton University Press

10 Bshary, R. and Bergmüller, R. (2007) Distinguishing four fundamental approaches to the evolution of helping. J. Evol. Biol. 21, 405-420

11 Alcock, J. (2005) Animal Behavior: An Evolutionary Approach, Sinauer Associates

12 Dugatkin, L.A. (2004) Principles of Animal Behavior, W.W. Norton

13 Enquist, M. and Ghirlanda, S. (2005) Neural Networks and Animal Behavior, Princeton University Press

14 Selten, R. (1991) Evolution, learning, and economic behavior. Games Econ. Behav. 3, 3-24

15 Binmore, K. (2006) Why do people cooperate? Polit. Philos. Econ. 5, 8196

16 Binmore, K. (2005) Natural Justice, Oxford University Press

17 Frank, S.A. (1998) Foundations of Social Evolution, Princeton University Press

18 Keller, L. (ed.) (1999) Levels of Selection, Princeton University Press

19 Bourke, A. and Franks, N. (1995) Social Evolution in Ants, Princeton University Press

20 Crespi, B.J. (2001) The evolution of social behavior in microorganisms. Trends Ecol. Evol. 16, 178-183

21 Michod, R.E. (1982) The theory of kin selection. Annu. Rev. Ecol. Syst. $13,23-55$

22 West, S.A. et al. (2007) Evolutionary explanations for cooperation. Curr. Biol. 17, 661-672

23 Lion, S. and van Baalen, M. (2007) Self-structuring in spatial evolutionary ecology. Ecol. Lett. 11, 277-295

24 Trivers, R.L. (1971) The evolution of reciprocal altruism. Q. Rev. Biol. $46,35-57$

25 Axelrod, R. and Hamilton, W.D. (1981) The evolution of cooperation. Science 211, 1390-1396

26 Luce, R.D. and Raiffa, H. (1957) Games and Decisions, John Wiley and Sons

27 Friedman, M. (1971) A non-cooperative equilibrium for supergames. Rev. Econ. Stud. 38, 1-12

28 Fudenberg, D. and Tirole, J. (1996) Game Theory, MIT Press
29 Axelrod, R. (1980) Effective choice in the prisoner's dilemma. J. Conflict Resolut. 24, 3-25

30 McElreath, R. and Boyd, R. (2007) Mathematical Models of Social Evolution: A Guide for the Perplexed, University of Chicago Press

31 Fudenberg, D. and Maskin, E. (1986) The folk theorem in repeated games with discounting or with incomplete information. Econometrica $54,533-554$

32 Osborne, J.M. and Rubinstein, A. (1994) A Course in Game Theory, MIT Press

33 Leimar, O. (1997) Repeated games: a state space approach. J. Theor. Biol. 184, 471-498

34 Binmore, K. et al. (1997) Evolutionary stability in alternating-offers bargaining games. J. Econ. Theory 80, 257-291

35 Roberts, G. and Sherrat, T.N. (1998) Development of cooperative relationship through increasing investment. Nature 9, 175-179

36 McNamara, J. (1999) Incorporating rules for responding into evolutionary games. Nature 401, 368-371

37 Ohtsuki, H. and Iwasa, Y. (2004) How should we define goodness? Reputation dynamics in indirect reciprocity. J. Theor. Biol. 231, 107-120

38 Nowak, M.A. and Sigmund, K. (2005) Evolution of indirect reciprocity. Nature 437, 1291-1298

39 Cavalli-Sforza, L.L. (1971) Similarities and dissimilarities of sociocultural and biological evolution. In Mathematics in the Archeological and Historical Sciences (Hodson, F.R. et al., eds), pp. 535-541, Edinburgh University Press

40 Cavalli-Sforza, L. and Feldman, M.W. (1981) Cultural Transmission and Evolution, Princeton University Press

41 Lumsden, C.J. and Wilson, E.O. (1981) Genes, Mind and Culture, Harvard University Press

42 Boyd, R. and Richerson, P.J. (1985) Culture and the Evolutionary Process, University of Chicago Press

43 Hofbauer, J. and Sigmund, K. (1998) Evolutionary Games and Population Dynamics, Cambridge University Press

44 Boyd, R.et al. (2003) The evolution of altruistic punishment. Proc. Natl. Acad. Sci. U. S. A. 100, 3531-3535

45 Hauert, C. and Doebeli, M. (2004) Spatial structure often inhibits the evolution of cooperation in the snowdrift game. Nature 428 , 643-646

46 Henrich, J. (2004) Cultural group selection, coevolutionary processes and large-scale cooperation. J. Econ. Behav. Organ. 53, 3-35

47 Ellison, G. and Fudenberg, D. (1993) Rules of thumb for social learning. J. Polit. Econ. 110, 612-643

48 Bergin, J. (2005) Microeconomics Theory: A Concise Course, Oxford University Press

49 Kandori, M. et al. (1993) Learning, mutation, and long run equilibria in games. Econometrica 61, 29-56

50 Young, H.P. (1993) The evolution of conventions. Econometrica 61,57-84

51 Binmore, K. et al. (1995) Musical chairs: modeling noisy evolution. Games Econ. Behav. 11, 1-35

52 Nowak, M. et al. (2000) Fairness versus reason in the ultimatum game. Science 289, 1773-1775

53 Hofbauer, J. and Sigmund, K. (2003) Evolutionary game dynamics. Bull. Am. Math. Soc. 40, 479-519

54 Boyd, R. and Richerson, P.J. (1982) Cultural transmission and the evolution of cooperative behavior. Hum. Ecol. 10, 325-351

55 Allison, P.D. (1991) Cultural relatedness under oblique and horizontal transmission. Ethol. Sociobiol. 13, 153-169

56 Henrich, J. and Boyd, R. (1998) The evolution of conformist transmission and the emergence of between-group differences. Evol. Hum. Behav. 19, 215-241

57 Boyd, R. and Richerson, P.J. (2005) The Origin and Evolution of Cultures, Oxford University Press

58 Fehr, E. and Fischbacher, U. (2003) The nature of human altruism. Nature 425, 785-791

59 Gintis, H. (2007) A framework for the unification of the behavioral sciences. Behav. Brain Sci. 30, 1-61

60 Lehmann, L. et al. (2008) Cultural transmission can inhibit the evolution of altruistic helping. Am. Nat. 172, 12-24

61 Lehmann, L. and Feldman, M.W. (2008) The co-evolution of culturally inherited altruistic helping and cultural transmission under random group formation. Theor. Popul. Biol. 73, 506-516

62 Harley, C.B. (1981) Learning the evolutionary stable strategy. $J$. Theor. Biol. 89, 611-633 
63 McNamara, J.M. and Houston, A.I. (1985) Optimal foraging and learning. J. Theor. Biol. 117, 231-249

64 Camerer, C. and Ho, T. (1999) Experience-weighted attraction learning in normal form game. Econometrica $67,827-874$

65 Giraldeau, L. and Caraco, T. (2000) Social Foraging Theory, Princeton University Press

66 Börger, T. and Rajiv, S. (1997) Learning through reinforcement and replicator dynamics. J. Econ. Theory 77, 1-14

67 Binmore, K. (1987) Modeling rational players: part 1. Econ. Philos. 3, 179-214

68 Binmore, K. (1988) Modeling rational players: part 2. Econ. Philos. 4, 9-55

69 Hanaki, N. et al. (2005) Learning strategies. J. Econ. Behav. Organ. 56, 523-542

70 Rubinstein, A. (1998) Modeling Bounded Rationality, MIT Press

71 Mailath, G. (1998) Do people play Nash equilibrium? Lessons from evolutionary game theory. J. Econ. Lit. 36, 1347-1374

72 Maynard-Smith, J. (1982) Evolution and the Theory of Games, Cambridge University Press

73 Borenstein, E. et al. (2008) Evolution of learning in fluctuating environments: when selection favors both social and exploratory individuals. Evolution Int. J. Org. Evolution 62, 586-602

$74 \mathrm{Kolb}, \mathrm{B}$. and Whishaw, I.Q. (2005) An Introduction to Brain and Behavior, Worth Publishers

75 Binmore, K. and Swierzbinski, J. (2006) A little behavioralism can go a long way. In Does Game Theory Work? The Bargaining Challenge (Binmore, K., ed.), pp. 257-276, MIT Press

76 Ledyard, J. (1995) Public goods: a survey of experimental research.. In The Handbook of Experimental Economics (Kagel, J. and Roth, A., eds), pp. 111-181, Princeton University Press
77 Sally, D. (1995) Conversation and cooperation in social dilemmas: a meta-analysis of experiments from 1958 to 1992. Rationality Soc. 7, 58-92

78 Andreoni, J. (1988) Why free ride? Strategies and learning in public goods experiments. J. Public Econ. 37, 291-304

79 Henrich, J. et al. (2005) 'Economic man' in cross-cultural perspective: behavioral experiments in 15 small-scale societies. Behav. Brain Sci. $28,795-855$

80 Gintis, H. (2003) Solving the puzzle of prosociality. Rationality Soc. 15 , $155-187$

81 Gintis, H. et al. (2003) Explaining altruistic behavior in humans. Evol. Hum. Behav. 24, 153-172

$82 \mathrm{Fehr}$, E. et al. (2002) Strong reciprocity, human cooperation, and the enforcement of social norms. Hum. Nat. 13, 1-25

83 Hagen, E.H. and Hammerstein, P. (2006) Game theory and human evolution: a critique of some recent interpretations of experimental games. Theor. Popul. Biol. 69, 339-348

84 Gale, J. et al. (1995) Learning to be imperfect: the ultimatum game. Games Econ. Behav. 8, 56-90

85 Crockett, M.J. et al. (2008) Serotonin modulates behavioral reactions to unfairness. Science 320,1739

86 Getty, T. (2007) In evolutionary games, enlightened self-interests are still ultimately self-interests. Behav. Brain Sci. 30, 25-26

87 Leimar, O. (2001) Evolutionary change and darwinian demons. Selection 2, 65-72

88 Richerson, P.J. and Boyd, R. (2005) Not by Genes Alone, University of Chicago Press

89 McFarland, D. and Houston, A. (1981) Quantitative Ethology: The State Space Approach, Pitman 